\title{
تشخيص وخزات الهتصاد السلوكي في خيارات الهفراد \\ دراسة استطلاعية لاراء عينة من الأساتذة والطلاب قسم الاقتصاد في كلية الادارة والاقتصاد
}

\author{
م. فان ميران شاهين، قسم الاقتصاد، جامعة نوروز، اقليم كودستان العراق
}

يعد الاقتصاد السلوكي أحدى الأدوات الحديثة لعلم الاقتصاد التي دجمت علم النفس المتغير مع الفرضيات الاقتصادية الجمردة الأمر الذي ساهم في تفسير الكثير من سلوكيات الأفراد الغير عقلانية التي

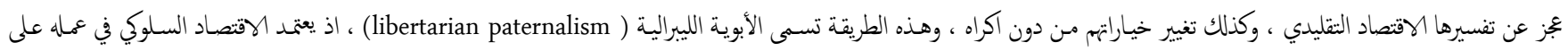

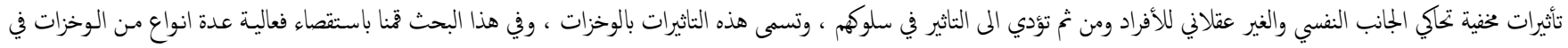

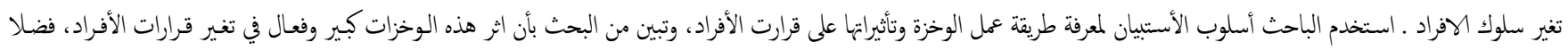

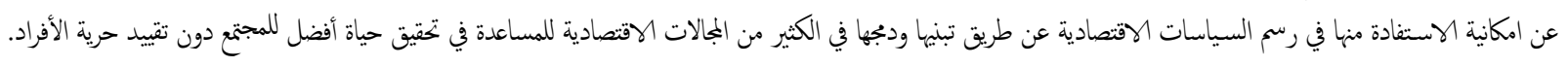
الكلمات الدالة: الاقتصاد السلوكي، الأبوية اليبرالية، الوخزة، السلوك الغير عقلاني، الاقتصاد الكلاسيكي.

1

الافراد دون اجبارهم او دون المساس بحريتهم الفردية التي يقدسها الاقتصاد العقلاني.

\section{1}

المشكلة التي تواجه الاقتصاد الكلاسيكي(التقليدي) هي عدم قدرته على تفسير ومن ثم تغير سلوكيات الافراد الغير عقلانية، وذلك بسب الفرضيات الغير واقعية التي يتمد عليها في تحليله، وهذه المشكلة أدت الى الكثير من التهيزات في خيارت الافراد مما اجبر الحكومات على التدخل عن طريق القوانين وادوات السياسة المالية والنقدية والتي تؤدي الى فقدان جزء من حرية الافراد التي أعطاه الاقتصاد للافراد والسوق، وايضاً تؤدي الى زيادة حجم تدخل الحكومات في الحياة

$$
\text { الاقتصادية وارتفاع أعبائها. }
$$

\section{1}

ان للاقتصاد السلوكي ووخزاته قدرة كبيرة على تفسير وتعديل الكثير من التحيزات في قرارات الافراد عن العقلانية وتغير سلوكمم نحو خيارات افضل دون التقليل

$$
\text { من حرياتهم او اجبار هم على خيارات معينة. }
$$

\section{1}

اعطاء فكرة عن تاريخ الاقتصاد السلوكي من خلال الدراسات السابقة توضيح مفهوم الاقتصاد السلوكي وهندسة الحيارات المرتبطة به.
شهدت نهاية عقد السبعينيات من القرن الماضي بداية الاهتام وتسليط الضوء

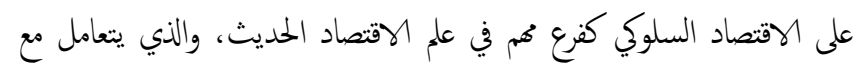
جانب عم من سلوك الأفراد وهو الجانب النفسي والني يصعب قياسه وتحليله، اذ يسعى الاقتصاد السلوكي من خلال استخدام الوخزات الى الثاثير على خيارات الافراد ومحاولة تغيرها نحو خيارات تقدم لمم حياة أفضل دون المساس او التقليل من حرياته، وهذا الامر يجتاج الى اعادة النظر في فرضية أساسية بنى علهيا الاقتصاد الكلاسيكي نظريته وهي ان الافراد سواء كنوا مستهلكين او منتجين يجب ان يتصرفوا بشكل عقلاني داخل السوق ومن ثخ سوف ثكون خياراتهم عقلانية وبالنتيجة سيعمل السوق بشكل كفوء، اما الاقتصاد السلوكي فانه يبني نظريته على فرضية اساسية اكثر واقعية وهي ان الافراد داخل السوق لا يتصرفون بشكل عقلاني، وان تحديد التصرف العقلاني من الغير عقلاني يجتاج الى معرفة وادراك واسع في علم النفس وهذا ما يؤكد عليه الاقتصاد السلوكي الني دمج علم النفس مع علم الاقتصاد.

1.1 بسب التطور السريع في الحياة الاقتصادية أصبح من المهم استخدام ادوات جديدة تساعدنا في التحليل الاقتصادي، من هنا برز دور الاقتصاد السلوكي في مي مئ تفسير الكثير من سلوكيات الافراد الغير عقلانية على اساس علمي واقتصادي،ان اهمية البحث تاتي من كونه يختبر قدرة الاقتصاد السلوكي على تغير خيارات 
ولكن هذه الفرضيات ساعدتنا على وضع نماذج رياضية وقياسية سهلت علينا تفسير الكثير من الظواهر الاقتصادية، ومن اهم الفرضيات التي يعتمد عليها الاقتصاد الكلاسيكي هي العقلانية (الرشادة) من قبل المستهاك والمنتج وهذه الفرضية يصعب تطبيقها في السوق بسب عدة عوامل اهها العامل السلوكي والنفسي النان يؤديان الى تحيزات كيرة في قراراتنا ومن ثخ خيارتنا ، ومن هنا ظهر الاقتصاد السلوكي لمحاولة فهم هذه التحيزات عن العقلانية (اي الغيرعقلانية) والاستفادة منها في تحسين قرارات الافراد نحو خيارات تقدم لم حياة افضل. والجدير بالذكر هنا ان السوق فيه خيارات متنوعة ومتشاهة في نفس الوقت، وان الاختيار لما يلائم الفرد منها تختاج الى وقت وبجد كير ، لذلك يعمل الاقتصاد

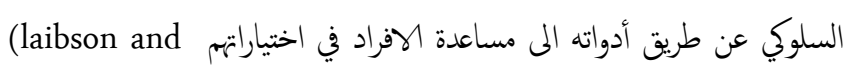
john,2015,18) في الحتيقة ان الاقتصاد السلوكي هو ليس بالضد من الاقتصاد التقليدي ولكنه يقوم بتعديل فرضيات الاقتصاد الكلاسكي بما يتلائم مع الدوافع الغيرعقلانية للفرد عن طريق استخدام الوخزات ، حيث ان الاقتصاد السلوكي مهتم بتفسير والتعامل مع الجانب الغير عقلاني للافراد من خلال اداة جديد تسمى الوخزة ،أضاف الاقتصاد السلوكي وبقوة علم النفس ومتغيراته الى علم الاقتصاد وبها طور اداة جديدة تضاف المى ادوات الاقتصاد في تحليل وتفسير سلوك الافراد والسوق وتوفير حياة افضل للمجمتع (Wilkinson and Klaes,2012,33) والسؤال الذي يككن ان يثار هنا هو ماهي الوخزة ؟ في الواقع ان الوخز لغةً: همس او حث او نخز بلطف شخص ما لجذب اهتمامه. اما تعريف الوخزة في سياق بحثنا هو عبارة عن اي حافز او تأثير خني يؤثر على نسية الفرد ومن ثم

$$
\text { يؤدي المى تغير في سلوكه وخيارته. }
$$

في الحتيقة ان السياسة الاقتصادية هدفها تحتيق الاستقرار الاقتصادي والاجتاعي وتقديم المنفعة العامة للمجتمع عن طريق استخدام اداوت السياسات الاقتصادية منها الضرائب والقيود التي توضع من خلال القوانين ومن ثم فان هذه الأدوات تحد وتقيد من حرية الافراد داخل السوق، فضلا عن تحميل الحكومة عبئ و تكاليف كبيرة من خلال ازدياد تدخلها في الحياة الاقتصادية، في حين ان سياسة الوخز التي يعتمدها الاقتصاد السلوكي تقلل من تدخل الحكومة في الحياة الاقتصادية ومن ثم الى تقليل الاعباء والنكليف على الحكومات وبالمقابل تعطي

$$
\text { مساحة اكبر من الحرية للافراد في اختيار خيارته. }
$$

اختبار فعالية أدوات الاقتصاد السلوكي ومساهمها في تغير سلوك وخيارات

الافراد.

بيان اهية الاقتصاد السلوكي في السياسات الاقتصادية

5.1

يتبع البحث المنجج الاستنبطي من خلال توضيح الفروقات بين الاقتصاد الكلاسيكي والاقتصاد السلوكي كنج عام اضافة الى استخدام طريقة الاستبيان

$$
\text { لتوضيح الية عمل الاقتصاد السلوكي كنج خاص. }
$$

2. المبحث الاول: تطور الاقتصاد السلوكي وعلاقته بالاقتصاد التقليدي

1.2 بداية الاقتصاد السلوكي والدراسات السابقة

بداء الأهتمام بالاقتصاد السلوكي في نهاية السبعينات من القرن الماضي، من خلال بكثين تم نشر الاول منها في سنة 1979 تحت عنوان (نظرية الاحتمالية:تحليل قرارات الافراد تحت ظروف الخاطرة) حيث تم نشره من قبل باحثين في علم النفس هـا( Tversky Daniel Kahneman and Amos في المجلة العلمية حيث بين الباحثين (prestigious and technical economic journal) كيفية تقييم الافراد لخياراتهم الحالية، وذلك عن طريق مقارنتها مع خيارات سابقة مشاهة للخيار الحالي، كما حلل البحث فكرة ان الافراد دائما ينفرون من الخسارة(loss-averse)، اي انهم ميالون الى الخخاطرة لتجنب الخسارة اكثر من ميلهم للمخاطرة للحفاظ على الارباح. اما البحث الثاني الذي سلط الضوء على الاقتصاد السلوكي كان تحت عنوان (نحو النظرية الايهابية في خيارات المستهلكين) حيث تم نشره سنة 1980 من قبل الباحث الاقتصادي(Richard Thaler) وبعدها اصبح الاقتصاد السلوكي معطة مهمة للبحث العلمي لعلماء النفس والاقتصاد. وفي عام 2008 قام ننس الاقتصادي (Richard Thaler) من قسم الاقتصاد و (Cass R. Suntein) من قسم القانون بكتابة بحث تحت عنوان ( Nudge: improving) decisions about health, wealth and happiness عichard Thaler الاقتصاد السلوكي ودوره في تخسين حياة الافراد والمجتم.

2.2 مفهوم الاقتصاد السلوكي بني الاقتصاد على اساس الفرضيات والتي كان بعضها غير واقي ولايمكن تطبيقه، 


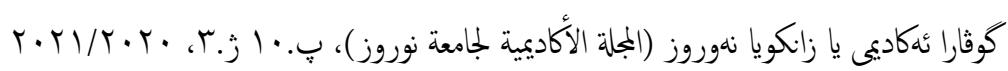

3. المبحث الثاني: الأبوية الليبرالية وهندسة الحيارات في ظل الاقتصاد السلوكي

1.3 الابوية الليبرالية libertarian paternalism

للوهلة الاولى يين لنا هذا المصطلح بانه يعاني من التناقض ، ولكن حقيقة الامر تشير الى انه مصطلح يحمل في طياته نمط جديد يساعد في الثاثير على خيارات الافراد ويغيرها نخو الأفضل مع الاحتفاظ بحريتهم في الاختيار ، وحسب ما يؤمن به الاقتصاد التقليدي فأن الافراد يقومون دائماً باخذ الخيار الذي يجعل حياتهم أفضل وذلك من خلال بجثم المستمر عن المنغعة والتي يتخذون كل قراراتهم في ضوئها مع اشتراط الحرية النامة لمم في خياراته ، وهذا ما يسى بالعقلانية او الرشادة الاقتصادية ، ولكن ما يحدث في الواقع ان عقلانية الافراد محدودة اي ان الكثير من خياراتم غير صائبة لأسباب كثيرة لا يسعنا ذكرها الان. في الواقع ان هذا يتطلب بأن يكون هناك تدخل في توجيه خيارات الافراد بطريقة خفية حتى لايشعرون بان حريتهم قد تأثرت ، والابوية الليبرالية لها وبجان الوول أبوي يفرض خيار محتوم والثاني يعطي الحرية في الحصول على الخيار الحمتوم من عدمه (Gruber,2001,18) الابوي الذي عادة ما يكون الافضل دون حضر الخيارات الاخرى، وعلى العكس من الادوات الابوية التقلدية مثل الغرامات والضرائب والدع والقوانين الصارمة التي تجبر الافراد على تغير خياراتهم نخو اخرى افضل دون اعطائهم الحرية في الاختيار، فأن الابوية المصاحبة مع الليبرالية تسهل الطريق امام العقلانية المحدودة لدى الافراد في موابحة خياراتهم المتعددة ، في واقع الامر ان الوضع الأفتزاضي(default option) التي تضعه الشركات بين خياراتها هو افضل مثال للابوية الليبرالية حيث يتم وضع الحيار الذي يقدم للافراد حياة افضل كخيار اولي موجود مسبقاً ومن ثم يستطيع الفرد تغير هذا الخيار ان اراد ذلك، فعلى سبيل المثال فان مدير الكلفنريا في مدرسة ابتدائية والمعني بترتيب المواد الغذائية بالطريقة التي تعزز الأكل الصحي هو ايضاً مثال عن هذا النمط الجديد ، ولكي نطبق الابوية الليبرالية بشكلها الصحيح نختاج الى تهندس للخيارات والذي يعمل على تريب الخيارات بطريقة تساعد الافراد على ان يختاروا منها ما يجعل حياتهم افضل في ظل العقلانية المحدودة. ( 2014,12, luoto)

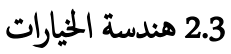

تعمل هندسة الخيارات على الثاثير على خيارات الافراد بالطريقة التي تجعل حياتهم افضل من خلال التعامل مع العقل اللاواعي للافراد ، ويعتمد أسلوب
من هنا فأن الاقتصاد السلوكي اذا تم تطبيقه في اليجتع بشكل ايجابي يؤدي الى رفع فعالية الحكومات في تحقيق اهدافها ليس من خلال ازدياد دورها في الحياة الاقتصادية ولكن من خلال رفع وتطوير كفائة ادواتها وقراراتها Thaler and .Sunstein,2008,52)

\section{2 الفرق بين الاقتصاد الكلاسيكي(التقليدي) والاقتصاد السلوكي}

هناك فروق جوهرية بين الاقتصاد الكلاسيكي والاقتصاد السلوكي، بالمقابل لا يككن الاستغناء عن الاقتصاد الكلاسيكي لانه على مدار قرون قدم لنا هذا الاقتصاد الكثير من الحلول لمشاكلنا الاقتصادية والاجتاعية "فالاقتصاد السلوكي"هو الاداة الاقتصادية التي تفسر لنا جزء من السلوك الغير عقلاني للافراد، والجدول رقٌ (1) يبين لنا الفروقات الجوهرية بين الاقتصاد التقليدي والسلوكي:

\section{جدول 1: الفرق بين الاقتصاد السلوكي والثقليدي}

\begin{tabular}{|c|c|}
\hline الاقتصاد السلوكي & الاقتصاد الكلاسيكي(التقليدي) \\
\hline يجب ان تكون فرضيات الاقتصاد & ليس بالضرورة ان تكون الفرضيات الذي \\
\hline السلوكي واقعية وقابلة للتطبيق في الحياة & يبنى عليها التحليل الاقتصادي واقعية. \\
\hline 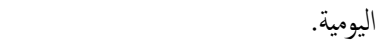 & \\
\hline 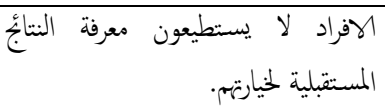 & لخافراد يستطيعون معرفة النتائج المستقبلية \\
\hline
\end{tabular}

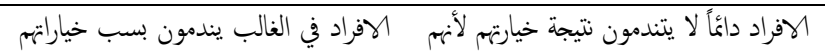

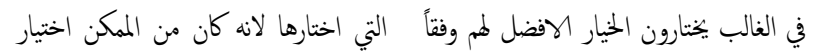

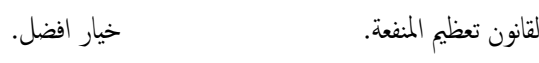

الافراد يمتلكون المعلومات والوقت الكافي الافراد لا يملكون المعلومات الكاملة

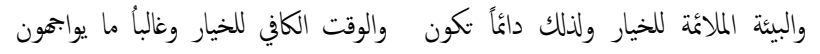
خيارته صائبة. بيئة غير ملائمة للختيار الخيار الافضل. الافراد والاسواق غالباً ما يعملون الافراد والاسواق في الغالب يعملون

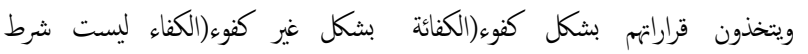

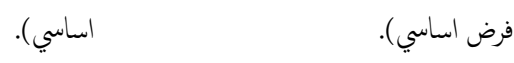

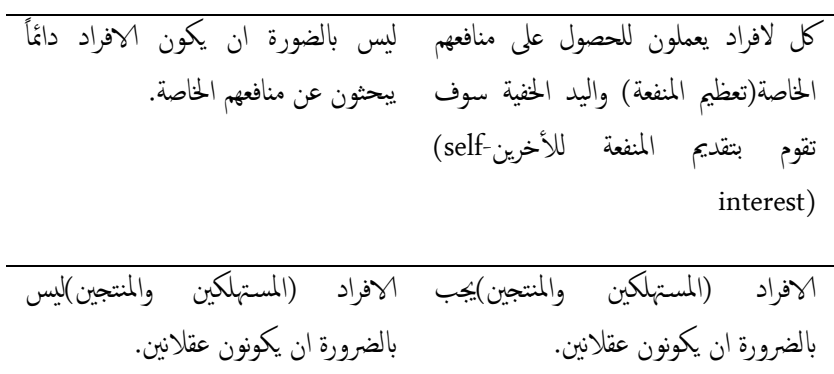
$\overline{\text { Source: Ariely ,Dan ,predictably irrational, HarperCollins Publishers, New York, }}$ 2008 


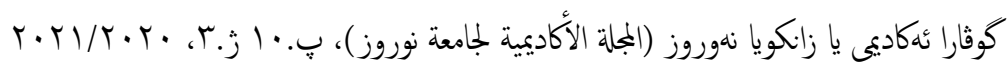

في الحتيقة يواجه الافراد الكثير من الخيارات في حياتهم اليومية، بعضها يكون

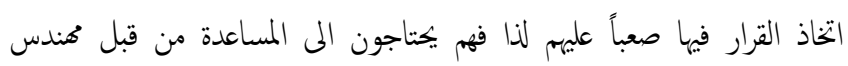
الخيارات، اذ ان هناك نوعان من الخيارات الاول يسمى الخيار الاستثمري وهذا النوع من تظهر كلفنه بشكل أني والفائدة تأتي لاحقاً مثال على هذا التيام بتنظيف الاسنان بشكل يوي أو خيار ممارسة الرياضة حيث أن هذه الحيارات تعد جيدة اذا تم اختيارها ولكن المشكلة فيها انها تحمل الفرد كلة أنية اي ان الفرد يجب ان ينفق الجهد والوقت في اتمام هذا الحيار بالمقابل فأن الفائدة من هذه الخيارات تمتاج الى وقت طويل لكي تظهر. النوع الثاني من الخيارات ريما نستطيع ان نسميها بالخيارات الرديئة أو الشريرة هذا النوع من الخيارت تظهر فائدتها او متعتها بشكل أني ومباشرة اما تكلفتها فتظهر في وقت لاحق ومثال ذلك شرب الكحول و التدخيين و اكل الشكولاته. وهنا يظهر دور هندس الحيارات الجيد في طريقة وضع الوخزات في النوعين من الحيارات الاستثمرية والشريرة لكي يحفز الافراد في الاستمرار في أختيار الحيار الاستثماري والابتعاد عن الخيار الشرير. (Boven,2009,23)

\section{4. المبحث الثالث: تطبيقات على ألية عمل الاقتصاد السلوكي}

1.4

من خلال النظرية الاقتصادية الجزئية يقوم الفرد بالمفاضة بين الحيارات وفقاً المنفعة المثأتية من استهلاكه للسلعة وبالثالي فانه يقوم بتوجيه انفاقه نخو السلعة التي تقدم له اكبر قدر من الاشباع، اي انها (النظرية الاقتصادية الجزئية) تفتزض

$$
\text { بأن المستهلك رشيد عقلاني. }
$$

بالمقابل فأن الاقتصاد السلوكي ينتقد هذه النظرية الاقتصادية من خلال التشكيك بعقلانية الفرد في السوق. معظم القرارات التي يتخذها الفرد داخل السوق هي قرارات غير عقلانية حسب وجه نظر الاقتصاد السلوكي، حيث تعتمد خيارات الفرد على الوخزة اكثر من اعتادها على المنفعة، هناك عدة انواع من الوخزات التي

$$
\text { تؤثز على خيارات الافراد منها: }
$$

\section{4 الحيارات السهلة نبياً تتغلب على الحيارت الصعبة}

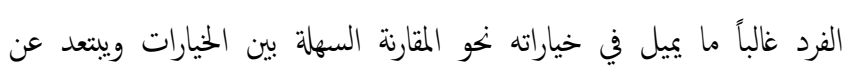
المقارنات الصعبة والمعقدة والتي تحتاج الى التفكير ، تم وضع بموعتين من الاسئلة لتوضيح هذه الفكرة, المجموعة الوولى تضم خيارين الحنيار الاول يحتوي على بجاز موبايل اي فون مستخدم السعر 250\$ والخيار الثاني يحتوي على موبايل اي رياي
هندسة خيارات الافراد على تسليط الضوء على خيارات معينة لتجذب اهتخام العقل اللاواعي للافراد ومن ثخ تحفيز هم على اختيارها ولكن دون المساس بحريته في الاختيار او حظر خيارات معينة. تنبع اهمية هذه الهندسة في ظل العقلانية المحدودة لدى الافراد في التعامل مع الكمية الكبيرة من الخيارات التي تواجهمه في حياتهم اليومية. يفترض الاقتصاد التقليدي ان قرارات الافراد دائماً تتحدد من خلال العقل الواعي تيوسي الذي عادة ما يمتاز بالرشادة الاقتصادية بما يتخذه من قرارات، ولكن في حقيقة الامر ان العقل اللاواعي لدى الفرد ايضاً يساهم وبشكل كبير في اتخاذ القرارات، والعقل اللاواعي لدى الأفراد لا يمناز في الكثير من الاحيان بالرشادة الاقتصادية لأعتبارات تتعلق بالعامل النفسي والبيئة التي نشاء بها عقل الفرد بالنتيجة فأن معظم قرارات الافراد ومن ثخ خياراتهم تفتقر الى الدقة في اختيار الصائب منها.

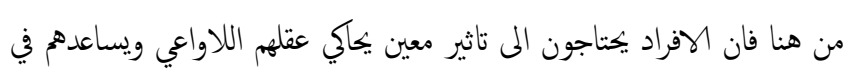
اتخاذ الخيار الصائب ، وهندسة الخيارات تعمل على ترتيب الخيارات بالطريقة التي تجذب فيها العقل اللاواعي للافراد.(Halpern,2016,17)

\section{3 دور الوخزة في هندسة خيارات الافراد}

تساعد الوخزة في عملية هندسة الخيارات بالطريقة التي تحول الغير عقلانية منها

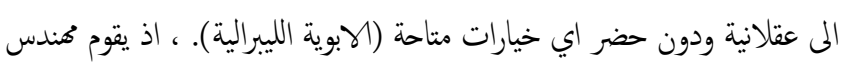
الخيارات بوضع حافز مخني أو ما يسمى بالوخزة بجيث لا يشعر بها الفرد في

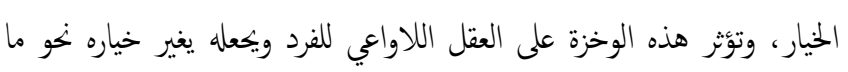
يريده هُندس الحيارات والذي من المفترض ان يكون هو الحيار الذي يقدم للفرد

الحياة الافضل.

وحسب المثال الذي تم ذكره سابقاً ملمير كافتريا المدرسة الابندائية فأنه يقوم بدور كهندس الخيارات الذي يقوم بعرض الطعام في الكافتريا بالطريقة التي تؤدي الى لى زيادة استهلاك الطعام الصحي بالنسبة للاطفال ومن ثم الى تحسين صحتهم، وهنا يقوم بوضع الطعام الصحي مثل الفواكه والطعام الذي يحتوي على نسبة عالية من الفيتامنات بمستوى نظر الاطفال وتسليط اضاءة قوية عليه ، بالمقابل يقوم هندس الخيارات بعرض الطعام الغير صحي مثل الحلويات والشكولاته في أماكن مرتفعة او بعيدة عن مستوى نظر الاطفال اذ ان ترتيب الطعام بها الشكل يعمل كوخزة في تمفيز الهطفال على استهلاك الطعام الصحي(Hossain,2012,122) 


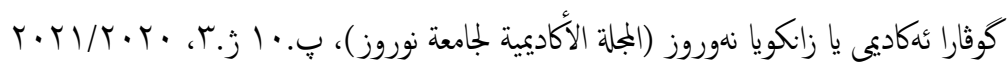

الخيار الارخص وهذا منطق مطابق لنظرية الاقتصادي الكلاسيكي الذي يبنى على عقلانية الفرد في التعامل مع الخيارات. في المقابل فأن الجمموعة الثانية من الخيارات كانت ننائجها بعيدة كل البعد عن العقلانية ،ماذا حصل لعقلانية الطلاب في المجموعة الثانية من الخيارات؟ قام الطلاب في المجموعة الثانية من الخيارات بالمقارنة بين ثلاثة خيارات، خيارين متشابهين(موبايل اي فون جديد السعر \$750 و موبايل اي فون جديد مع ساعة الاذن السعر \$750) وخيار مختلف(موبايل اي فون مستخدم السعر 250) عقل الفرد يميل الى الأختيار والمفاضلة بين الخيارات المتقاربة والسهلة والغير معقدة ولأبتعاد عن المقارنات المختلفة والصعبة بين الخيارات،من هنا قام الطلاب بأستبعاد الخيار الصعب وهو اي فون مستخدم السعر 250\$ والمقارنة بين خيارات سهلة التفضيل فيا بنيهم وهما موبايل اي فون جديد السعر \$750 و موبايل اي فون جديد مع سماعة الاذن السعر \$750 فبالتأكد سوف يميل الطلاب نخو الخيار المزي برأهه وهو موبايل اي فون جديد مع سماعة الاذن السعر \$750، وقد لعب هنا موبايل اي فون جديد السعر \$750 دور الوخزة لتغير خيارات الطلاب نخو خيار كانت قبل اسبوع خيار غير عقلاني. تحيز الافراد نحو الخيارات السهلة يككن ان تستخدم كاحدى ادوات المالية العامة المهمة التي تساعد في رسم السياسة الاقتصادية والمالية للحكومة وتغير سلوك الافراد من خيارات تضر بالمجتع والبيئة المى خيارات افضل.

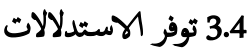

الفيضانات والزلازل كوارث طبيعة خطيرة ولكن أحتالية حدوثا في العراق منخفضة الى حد ما، بالمقابل حوادث السيارات والسرقة تعتبر اقل خطورة من الكوارث الطبعية ولكن احتالية حدوثها في العراق اكبر، من هنا فأن مخاوف الافراد تكون اكبر من الحوادث التي تكون احتالية حدوثها اكبر لانها تكون راسنة في العقل الباطن للفرد ومن ثم فان الفرد يحدد خياراته وفقاً لهذه المخاوف اكثر من تحديد خيارته على اساس العقلانية التي تنادي بها النظرية الاقتصادية

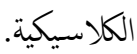
فالخيارات التي تحوم حولها مخاوف من احتالية حدوث كيرة او قد تم اختبار هذه المخاوف من قبل شخص اخر، يكون لها حظ اوفر في اختيارها. ومن خلال استثمار هذه الفكرة في سؤالين عن التأمين لها احتالية حدوث مختلفة طبقت على 50 طالب من طلاب قسم الاقتصاد، والسؤالين ها:فون جديد مع سمة الاذن السعر \$750 تم توجيه هذا السؤال على 80 طالب في جامعة نوروز وطلب منهم اختيار احدى الحيارين فكانت النتائُ كما يلي: جدول 2: يوضح الخيارات السهلة نسياً تتلب على الحيارت الصعبة

\begin{tabular}{|c|c|c|}
\hline & & المجموعة الاولى \\
\hline 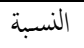 & 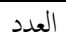 & الخيار \\
\hline$\% 86$ & 69 & جهاز موبايل اي فون مستخدم السعر \$250 \\
\hline$\% 14$ & 11 & م750 \\
\hline$\% 100$ & 81 & المجموع \\
\hline
\end{tabular}

من خلال الجدول اعلاه يتضح لنا بأن أغلب الطلاب قامو بأختيار الحنيار الرخيص حيث ان 86\% قد اختارو اي فون مستخدم السعر 250\$ بالمقابل 14\% اختارو اي فون جديد مع سماعة الاذن السعر \$750 حيث قام الطلاب بالمقارنة بين الحيار الاول والحنيار الثاني ثم وجدو اغلهم ان الخيار الوول هو مجزي اكثر بسب سعره المنخفض مقارنة مع الحيار الثاني، هذا التحليل يطابق مفهوم عقلانية الفرد في التصرف.بعد اسبوع واحد قُنا بتوزيع المجموعة الثانية من الحيارات على نفس الطلاب حيث كانت النتائُ ختنافة تمامأ، تم اضافة خيار ثالث على الخيارين السابقين وهو خيار اي فون جديد السعر \$750 فكانت النتأُجما موضح في الجدول ادناه

جدول 3: الميارات السهلة نسياً تتغلب على الخيارت الصعبة مع وجود الوخزة

\begin{tabular}{|c|c|c|}
\hline & & المجموعة الثانية \\
\hline 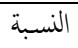 & 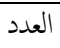 & الخيار \\
\hline$\% 10$ & 8 & موبايل اي فون مستخدم السعر \$250 \\
\hline$\% 15$ & 12 & موبايل اي فون جديد السعر \$750 \\
\hline \multirow[t]{2}{*}{$\% 75$} & \multirow[t]{2}{*}{61} & موبايل اي فون جديد مع سماعة الاذن السعر \\
\hline & & $\$ 750$ \\
\hline$\% 100$ & 81 & البمموع \\
\hline
\end{tabular}

نلاحظ من الجدول اعلاه ان النسب قد اختلفت ولصاح خيار اي فون جديد مع ساعة الاذن السعر \$750 حيث ارتفع نسبة الطلاب الذين اختارو هذا الحنيار من 14\% الى 75\% وانخفض اختيار الطلاب لخيار اي فون مستخدم السعر 250 من 86\% الى 10\% ،ماهو السبب في تغير تفضلات الطلاب خلال مدة زمنية قصيرة؟ السبب هو الوخزة التي تم وضعها في المجموعة الثانية من الخيارات وهي اي فون جديد السعر \$750\$. في الجمموعة الاولى من الخيارات كانت تختوي على خيارين واضحين وسهلين للطلاب فأستبعدو الخيار الاغلى نسبياً واختارو 


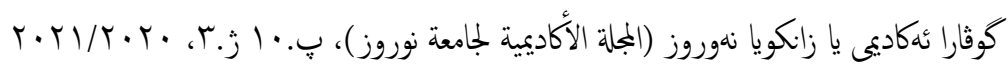

جدول 6: سوال (ب) الوخزة القوية

\begin{tabular}{|c|c|c|c|c|}
\hline الجموع & ريما & ل & نعم & الوخزة \\
\hline 51 & 7 & 11 & 33 & 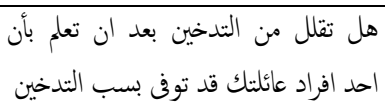 \\
\hline$\% 100$ & $\% 13$ & $\% 21$ & $\% 64$ & النسبة \\
\hline
\end{tabular}

من خلال سؤال (أ) الذي يوضح الوخزة الضعيفة يتين بأن 50\% من الطلاب لم يوافق على التقليل من التدخين وذلك بسب ان الوخزة لم تؤثر على سوك الفرد، اما السؤال (ب) فهو نفس سؤال (أ) ولكن الوخزة قوية ( متعلقة بمخاوف الموت) ،ووفقاً لسؤال (ب) فأن 64\% من الطلاب المدخنين اثرت عليهم هذه الوخزة

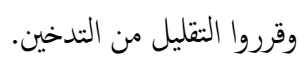

هذا السلوك الغير عقلاني للأفراد يوضح بأن خيارات الافراد تتاثر بعوامل نفسية اكثز من تأثرها بعوامل عقلانية، فأن قوة الوخزة هي التي تؤدي الى تغير سلوك

الافراد وخياراته.

من هنا فأن الحكومات مككن ان تستفاد من هذا النوع من الوخزات في عملية هندسة خيارات الافراد بالاتجاه الذي يحسن المستوى الاقتصادي والصحي

والاجتاعي لم.

\section{4 الحوف من الخسارة لها تأثير اكبر من متعة الريح على سلوك الافراد:}

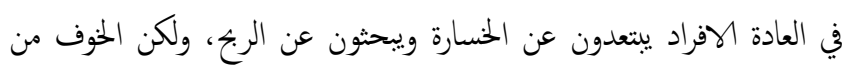
خسارة شيء تملكه في اغلب الاحيان يعادل ضعف متعة الفوز بنفس الشيء(وخزة؟) ، ومن ثم فأن الخوف من الخسارة يؤثر على قرارات الافراد اكثر من متعة الريح ، وهذه الوخزة تعمل على تثثيت الافراد على بعض الخيارات

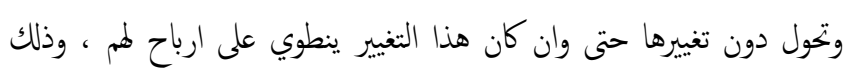

$$
\text { بسب وخزة الحوف من الخسارة التي تتغلب على متعة الربح. }
$$

وعلى هذا الاساس قام الباحث بوضع سؤال افتراضي يوضح هذه الوخزة وتم توزيعه على 50 تدريسي وموظف في كلية الادارة والاقتصاد في جامعة نوروز ،

حيث كان السؤال على الشكل التالي:

أذا كان لديك \$ \$ \$أردت ان تستثرها في البورصة واذا علمت ان احتالية

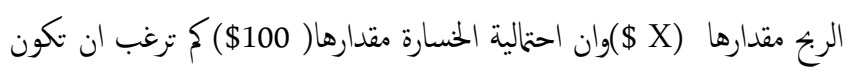
قيمة (X) لكي تقوم بالأستثار؟ وكانت الخيارات الى (X) هي : \$ \$ \$100-

$$
\text { وكانت الاجابات على النحو الاتي : }
$$

السؤال الاول: هل تفضل التأمين على السيارة بقسط شهري 100\$ ؟

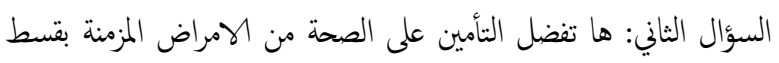

\begin{tabular}{|c|c|c|}
\hline 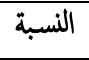 & 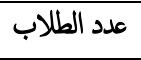 & نوع التأمين \\
\hline$\% 66$ & 33 & التأمين على السيارة القسط الشهري 100\$ \\
\hline \multirow[t]{2}{*}{$\% 34$} & 17 & التأمين على الصحة من الامراض المزمنة القسط \\
\hline & & الشهري \$50\$ \\
\hline \%100 & 50 & المجموع \\
\hline
\end{tabular}

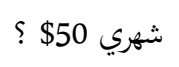

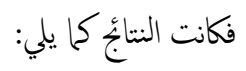

\section{جدول 4: وخزة توفر الاستدلالات}

من الجدول اعلاه يكن ان نستنتج انه بالرغ من ان التأمين على الصحة من الامراض المزمنة اهم واقل سعراً من التأمين على السيارة ولكن 66\% من بـ الطلاب قررو الذهاب مع التأمين على السيارة بالمقابل 34\% من الطلاب اختارو الخيار الثاني وهو التأمين على الصحة من الامراض المزمنة بسب ان احتالية تكرار حوداث السيارة اكثر ومن ثم فأن احتالية تكرار هذه الخخاوف تعمل كوخزة تؤدي الى تغير سلوك الافراد نو خيارات معينة. من هنا فأن هذه الوخزة يككن ان يتم الاستفادة منها في رسم السياسات الاقتصادية والمالية بالشكل الذي يساهم في توزيع الموارد الاقتصادية بصورة تتلائم مع مخاوف الافراد من الحوادث الاكثر تكررا ، اكثر من اعتادها على الخخاوف

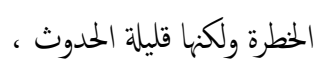
وفي هذه الاطار يمكن ان تلعب قوة الوخزة دور كير في تغير سلوك الافراد اذ كلما كانت المخاوف والعقبات التي تحوم حول الحيار كبيرة وراسخة في عقل الفرد كلما كان عملت الوخزة بشكل اكثر فعالية. الاختبار الاخر الذي تم من خلاله فص اثر الاستدلالات كان عن طريق توزيع نوين من الاسئة(أ،ب) ، على 51 طالب مدخن في قسم الاقتصاد في جامعة

\begin{tabular}{|c|c|c|c|c|}
\hline الجمموع & ريما & لا & نعم & الوخزة \\
\hline 51 & 15 & 26 & 10 & 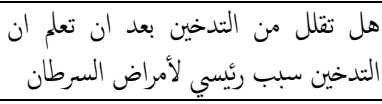 \\
\hline$\% 100$ & $\% 29$ & $\% 50$ & $\% 19$ & النسبة \\
\hline
\end{tabular}

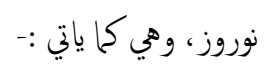
السؤال (أ) يجتوي على سؤال فيه وخزة ضعيفة والسؤال (ب) فيها وخزة قوية

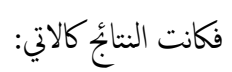

جدول 5: سؤال (أ) الوخزة الضعيفة 


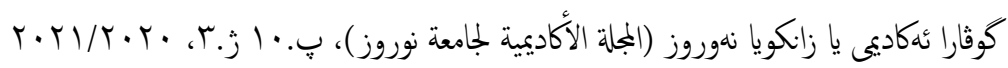

\begin{tabular}{|c|c|}
\hline \multicolumn{2}{|c|}{ جدول 8: وخزة التبيت } \\
\hline التقدير & السؤال الاول \\
\hline 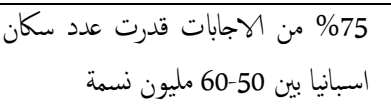 & هل يمة، يفوق عقدر عدد سكان اسبانيا؟ اسبانيا 40 مليون \\
\hline
\end{tabular}

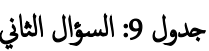

\begin{tabular}{|c|c|}
\hline التقدير & السؤال الثاني \\
\hline 82\% من الاجابات قدرت عدد سكان & هل يفوق عدد سكان اسبانيا 60 مليون \\
\hline اسبانيا بين 70-85 مليون نسمة & نسمة،ك تقدر عدد سكان اسبانيا؟ \\
\hline
\end{tabular}

من خلال جدول رڤ(7) الذي يبين الوخزة المنخفضة (40 مليون نسمة) ، قدر 75\% من الطلاب عدد سكان اسبانيا بين 50 الى 60 مليون نسمة ، في حين جدول رقً (8) الذي فيه وخزة مرتفعة (60 مليون) والذي تم من خلالها تثبيت معلومة لدى الطلاب ينطلقون من خلالها لكي يقدروا عدد سكان اسبانيا حيث قدر 82\% من 25 طالب عدد سكان اسبانيا بين 70-85 مليون نسمة، بالنتيجة اعتمد الطلاب على وخزة التثبيت في تعديل قراراتهم،اي ان هذه الوخزة يككن ان تستخدم لتغير سلوك الافراد نخو خيارات جيدة مثال على ذلك تسخدم كثير من الدول المتقدمة هذه الوخزة لترشيد استهلاك الماء او الكهرباء من خلال وضع تعليمات توضح نسبة استهلاك الماء مثلاً في الحي المجاور هذه يحفزهم على عدم

$$
\text { تجاوز هذه النسبة. }
$$

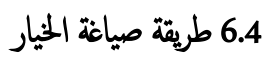

هنالك فجوة في اللغة بين الحكومة والناس تجعل الناس يرفضون ما تقوله الحكومة، ولكن إذا صيخ القرار نفسه بلغة مقبولة ومطمئنة يصبح القبول به أكثر يسراً ومن ثم فان طريقة صياغة الحيار تعمل كوخزة في تغير سلوك الافراد نخو خيارات معينة.فكلما كانت صياغة الخيار بشكل ايجابي كان الناثير على سلوك الفرد بشكل اكبر. وعلى هذا الاساس قمنا باجراء اختبار يوضح هذه الوخزة من خلال سؤالين تم صياغتها بشكلين مختلفين، كل سؤال تم توزيعه على 50 طالب من طلاب

$$
\text { كلية الادارة والاقتصاد في جامعة نوروز · }
$$

السؤال الاول : يسأل الطالب عن رغبته بأجراء عملية طبية معينة اذا قال له

$$
\text { الطبيب ان نسبة فشل العملية تقدر 9\% . }
$$

السؤال الثاني كان على الشكل الاتي: هل ترغب باجراء العملية نفسها اذا قال

$$
\text { لك الطبيب ان نسبة النجاح تقدر 91\% ؟ وكانت النتائُ على النحو التالي: }
$$

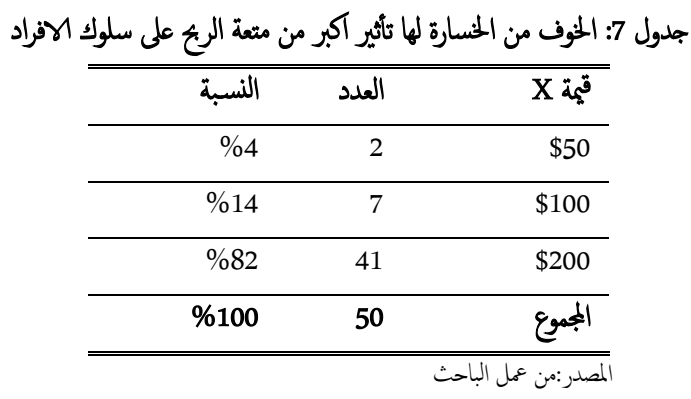

حيث يتبين من الجدول اعلاه ان 4\% من الاجابات اختارت 50 \$بح لكي ري لي تعادل \$100\$ خسارة في حين ان 14\% من الاجابات اختارت ان تساوي قيمة الربح مع قيمة الخسارة، بالمقابل 82\% من الاجابات اختارت ان تكون قيمة الربح تساوي \$200 ، ماذا يعني هذا؟ هذا يعني ان 100\$ خسارة تعادل \$200 ربح وهذا غير منطقي من وبجة نظر الاقتصاد الكلاسيكي ولكن بسب وخزة الخوف من الخنارة التي تتغلب على متعة الربح التي تؤكد عليها نظرية الاقتصاد السلوكي تصبح هذه الروئية منطقية ، وهذا يؤكد فرضية أن الخوف من فقدان شيء يملكه الفرد تعادل ضعف متعة الفوز

$$
\text { بنفس الشيء. }
$$

بالنتيجة يمكن ان تستخدم هذه الوخزة في السياسات الاقتصادية والمالية لتحفيز الافراد على عدم تغير خياراتهم الحالية وابعادهم عن الخيارات الاخرى.

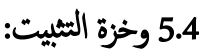

يعتمد الافراد في بعض الاحيان على نقطة معينة أو معلومة سابقة ومن خلالها ينطلقون نحو تحديد خيارته ولهذا تسمى هذه الوخزة وخزة التثبيت والتعديل. يمكن توضيح مفهوم وخزة التثبيت والتعديل بشكل افضل من خلال سؤالين فيها وخزين مختلفتين قمنا بتوزيع كل سؤال على 25 طالب من طلاب قسم الاقتصاد

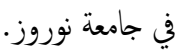
السؤال الاول: هل يفوق عدد سكان اسبانيا 40 مليون نسمة ؟ ماهو تقديرك لعدد سكان اسبانيا ؟ وضعنا في هذه السؤال نقطة ينطلق منها الطالب لتقديرعدد سكان اسبانيا وهي 40 مليون نسمة والتي تمثل وخزة تساعد في التقدير. السؤال الثاني:هل يفوق عدد سكان اسبانيا 40 مليون نسمة ؟ ماهو تقديرك لعدد سكان اسبانيا ؟ هنا في السؤال الثاني قنا بتغير الوخزة الى 60 مليون

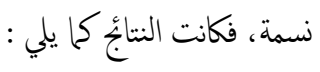




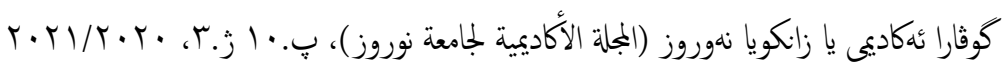

يتعامل الاقتصاد السلوكي مع الحنيارات من خلال الجانب النفي للأفراد. لا يحد الاقتصاد السلوكي من حريات الافراد في تحديد خياراتهم ولكنه يحاول ابعادهم عن الخيارات الرديئة والثريرة. الوخزات تعمل على الرفع من كفائة السياسات الحكومية في التعامل مع

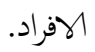

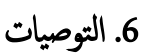

أعادة النظر في رسم السياسات الاقتصادية والاجتماعية بما يتلائم مع الجانب النفي المتغير للأفراد وذلك عن طريق

أستخدام تأثيرات الاقتصاد السلوكي من خلال تبني الحكومات طريقة الوخز الذي يؤكد عليه الاقتصاد السلوكي في تغير خيارات الأفراد الى لي لئري خيارات تقدم لمم حياة أفضل 2-اهتمام الحكومات بموضوع هندسة الخيارات وادراج هذا النوع من الهندسة في مناجه العلوم النفسية والعلوم الاقتصادية لكي يزداد وعي الافراد بمفهوم الاقتصاد السلوكي واداوته. 3-استخدام طريقة الوخز في السياسات الاقتصادية وذلك لتاثيرها على العقل الاواعي للافراد اكثر من القيود والقوانين والغرامات. 4-4الابتعاد عن التشريعات والقوانين القاسية في تغير سلوك الافراد لانها تقلل من حرياتهم في الاختيار بالمقابل استخدام تطبيقات الاقتصاد السلوكي في تحفيز الافراد في اختيار خيارات تقدم لمم حياة افضل.

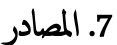

1. Laibson, David, and John A. list, Principles of (Behavioral) Economics.", American Economic Review 105 (5) (May), 2015

2. Wilkinson, Nick and Matthias Klaes, An Introduction to Behavioral Economics, $2^{\text {nd }}$ edition, Palgrave Macmillan, uk, 2012

3. Thaler, Richard H. and Cass R. Sunstein, NUDGE Improving Decisions About Health, Wealth, and Happiness, Yale University Press New Haven \& London, 2008

4. Ariely, Dan, predictably irrational, Harper Collins Publishers, New York, 2008.

5. Kahneman, Daniel, and Amos Tversky, "Prospect Theory: An Analysis of Decision under Risk.” Econometrica 47 (2), 1979.

6. Thaler, Richard H. "Toward a Positive Theory of Consumer Choice." Journal of Economic Behavior and Organization 1 (1): 1980.
جدول 10: جدول يوضح طريقة صياغة الخيار

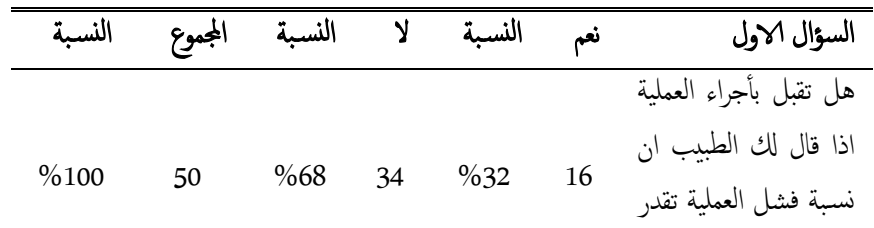

بي

المصدر:من عمل الباحث

جدول 11: طريقة صياغة الحيار مع وجود الوخزة

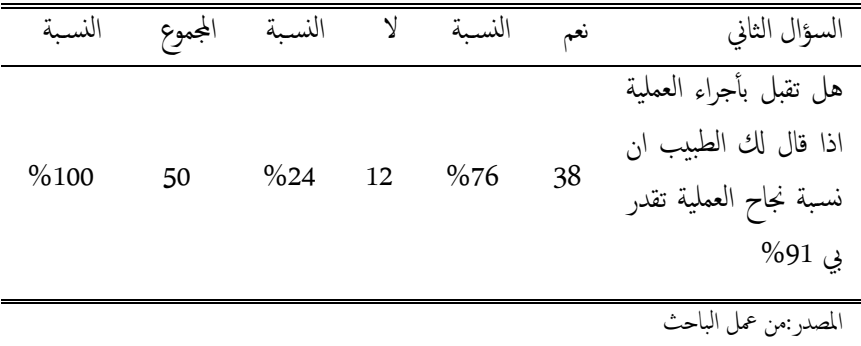

من خلال الجدولين اعلاه يتبين لنا بأن صياغة السؤال بشكل ايجابي مككن ان يغير في سلوك الافراد حيث ان 68\% من الطلاب قررو عدم اجراء العملية بسب احتالية فشلها التي تقدر بي 9\% في حين ان 76\% من الطلاب قررو اجراء العملية بسب احتالية نجاحا المرتفعة التي تقدر بي 91\%، في الحالتين فان نسبة النجاح والفشل هي نفها ولم تتغير ولكن طريقة صياغة السؤال هي التي تغيرت وبالتالي اثرت على خيارات الافراد هذا ايضاً يعتبر سلوك غير عقلاني من وجه ترئ نظر الاقتصاد الكلاسيكي ولكن يعتبر امراً طبيعياً من منطلق الاقتصاد السلوكي الذي يعتمد على الوخز اكثر من اعتماده على العقلانية في تحليل سلوك الافراد. هناك تاثيرات اخرى للتعبير عن عمل الاقتصاد السلوكي عن طريق الوخز مثل تحيز الفرد الى الوضع الراهن او مايسمى التهيز للخيار الافتزاضي حيث يميل الافراد الى الابقاء على الخيار الافتراضي الذي تم تحديده لمم مسبقاً دون تغير تهري ولكن سوف ككتني في هذا البحث للوخزات التي تح شرحا بشكل مفصل اعلاه.

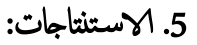

من خلال الاختبارات التي تم اجرائها حول هندسة الخيارات والسلوك الاقتصادي توصل البحث الى عدد من الاستناجات وهي كما ياتي : الاقتصاد السلوكي لا يعتبرعلماً منفصلاً عن علم الاقتصاد ولكنه اداة تضاف الى أدوات علم الاقتصاد التي تساهم في تفسير سلوكيات المستهلكين والمنتجين الغير عقلانية داخل السوق. يعمل الاقتصاد السلوكي من خلال الوخزات وهي تاثيرات مخية في

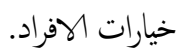




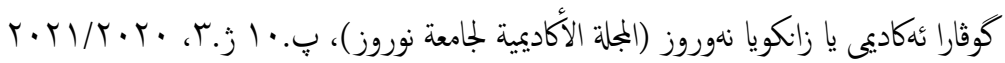

7. Uoto, Jill and Katherine Carmen, behavioral economic guidelines with application for health interventions, inter-american development bank, 2014.

8. Halpern D., inside the nudge unit: how small changes can make a big difference, random house, 2016.

9. Boven 1, the ethics of nudge, preference change, springer, Dordrecht, 2009.

10. Gruber, Jonathan, and Botond Ko" szegi. "Is Addiction 'Rational'? Theory and Evidence." Quarterly Journal of Economics 116 (4), 2001.

11. Hossain, Tanjim, and John A. List. "The Behavioralist Visits the Factory: Increasing Productivity Using Simple Framing Manipulations, 2012. 